\title{
In Vitro Evaluation of the Effect of Different Surface Treatments of a Hybrid Ceramic on the Microtensile Bond Strength to a Luting Resin Cement
}

\author{
Fariba Motevasselian ${ }^{1 *(1)}$, Zahra Amiri' ${ }^{2}$ Nasim Chiniforush ${ }^{3}$, Mansoreh Mirzaei ${ }^{1}$, Van Thompson ${ }^{4}$ \\ ${ }^{1}$ Restorative and Esthetic Dentistry Department, School of Dentistry, Tehran University of Medical Sciences, Tehran, Iran \\ ${ }^{2}$ Department of Restorative Dentistry, School of Dentistry, Semnan University of Medical Sciences, Semnan, Iran \\ ${ }^{3}$ Laser Research Center of Dentistry, Dentistry Research Institute, Tehran University of Medical Sciences, Tehran, Iran \\ ${ }^{4}$ Tissue Engineering and Biophotonics, King's College London Dental Institute
}

\section{*Correspondence to \\ Fariba Motevasselian, Assistant professor, Tehran University of medical Sciences, School of \\ Dentistry, Restorative and Dentistry Esthetic Department. \\ Tel: + 9821-88015801-88015088 Email: \\ f-motevasselian@sina.tums.ac.ir, fariba.motevasselian@yahoo.com}

Published online October 1, 2019

\begin{abstract}
Introduction: The aim of the present study was to investigate the effect of different surface treatments of a hybrid ceramic, Vita Enamic, on the micro-tensile bond strength ( $\mu$-TBS) to resin cement.

Methods: Ten blocks $(3 \times 10 \times 8 \mathrm{~mm})$ were retrieved from the original blocks and divided into 5 groups according to the different surface treatments performed: Groups 1:35\% acid phosphoric for 60 seconds (PA); group 2: Sandblasting with $50 \mu \mathrm{m} \mathrm{Al} \mathrm{O}_{3}$ particles for 10 seconds (SB); groups 3: 9.5\% hydrofluoric acid for 60 seconds (HF), group 4: The Er:YAG laser $(2 \mathrm{~W}, 10 \mathrm{~Hz})$ (ER1), group 5: The Er:YAG laser $(3 \mathrm{~W}, 10 \mathrm{~Hz})(E R 2)$. All treated surfaces were salinized and the blocks with similar surface treatments were bonded together using a dual-cured resin cement and light-cured. After 24-hour storage in water, the blocks were cut into beams $\left(1 \mathrm{~mm}^{2}\right)$. Half of the specimens in each group $(n=16)$ were tested immediately and the rest were subjected to thermocycling between $5^{\circ} \mathrm{C}$ and $55^{\circ} \mathrm{C}$ for 6000 cycles before the $\mu$-TBS test at a crosshead speed of $0.5 \mathrm{~mm} / \mathrm{min}$. The data were analyzed using two-way analysis of variance (ANOVA) and Tukey HSD tests and the significance level was set at 0.05. The failure mode was evaluated by using a stereomicroscope.

Results: The $\mu$-TBS was clearly influenced by surface treatment methods $(P<0.001)$ and thermocycling significantly decreased the bond strength values in all groups $(P=0.007)$. The highest value (66.07 $\mathrm{MPa} \pm 11.3$ ) was obtained for the HF groups with no thermocycling and the lowest values were observed in the laser groups with no significant difference among different irradiation parameters. Adhesive failure was mainly observed in the PA and SB groups while mixed failure was predominantly shown in the laser and HF groups.

Conclusion: This study demonstrated that surface treatment of VE with HF and salinization could improve the bond strength to a dual-cured resin cement, and Er:YAG laser irradiation with the evaluated parameters did not promote the adhesion of the resin cement to VE.
\end{abstract}

Keywords: Er:YAG laser; Hybrid ceramic; Tensile strength; Surface treatment.

\section{Introduction}

Ceramics and indirect composite restorations are two classes of restorative materials which have been widely used recently due to their esthetic properties. Dental ceramics are inorganic materials usually made up of crystalline and glassy phases. ${ }^{1}$ Although dental porcelain has natural tooth appearance, there are some inherent problems associated with dental ceramics, like potential abrasivity to the opposing dentition due to their high hardness and chipping owning to their brittleness. ${ }^{2}$ Dental resin composites are comprised of an organic resin matrix mixed with reinforcing inorganic filler particles. Composites are less brittle and do not cause high antagonistic tooth wear. However, the wear of the material itself is higher and has lower color stability which limit their use. ${ }^{3}$

Dental ceramics display some physical properties similar to those of human enamel, whereas composite resins properties are more comparable to dentin characteristics. ${ }^{4}$ There has been a need for a material that combines the advantages of ceramics with those of composites. ${ }^{4,5}$ The recently introduced polymer-infiltrated-ceramic-network or hybrid ceramic offers a combination of ceramic and polymer properties. ${ }^{5,6}$ 
Resin bonding is a critical step in the process of placing indirect restorations that rely on adhesive cementation to ensure proper retention, durability, and clinical performance. ${ }^{7}$ Therefore, the adhesive bond between different materials has to be adapted according to the physical/chemical characteristics of each material. Nondestructive methods for treating indirect restoration surfaces to enhance adhesion to resin luting cement are desirable. ${ }^{8,9}$

Different types of surface treatments have been used to improve the bond strength of resin cement to hybrid ceramic materials. Several of these techniques such as acid etching with hydrofluoric acid and air-particle abrasion systems ${ }^{9-12}$ are applied to increase the surface roughness of the materials. Recently, with laser technology advancement, the laser is used as a surface treatment method for indirect restorations including dental ceramics to improve their bonding to resin cement. ${ }^{13,14}$ It has been speculated that a laser beam causes surface alterations through ablation and melting of ceramic structure and has a roughening capacity of the inner surfaces of all-ceramics for adhesive luting procedures. ${ }^{13,15}$ Among the various type of lasers, Er: YAG is one of the most promising lasers due to its wavelength match with the absorption peak of water. ${ }^{16}$ The overheated water abruptly vaporizes and it causes the surrounding material to be exploded away in a thermomechanical ablation process. ${ }^{13,16}$ However, there is little in the existing literature about the laser treatment and its roughening capacity of hybrid ceramics. ${ }^{17}$

Measuring the bond strength of adhesive systems to a substrate is one of the methods to evaluate the effectiveness of surface treatment. However, restorations are exposed to chemical, thermal, and mechanical challenges in the oral environment which could have a weakening effect on the bond strength and the adhesive interface. ${ }^{18}$ Therefore, it is important to simulate these conditions in in vitro studies. Although several studies have reported different types of surface treatments applied to hybrid ceramics, ${ }^{9-11}$ there is no consensus on the most favorable surface treatment to strengthen bonding between resin cement and hybrid ceramics. The present study was, therefore, carried out to evaluate the effects of different surface treatments on the micro-tensile bond strength ( $\mu$-TBS) of an indirect CAD/ CAM hybrid ceramic, Vita Enamic (VITA Zahnfabrik, Bad Sackingen, Germany) to an adhesive luting resin cement with and without thermo-cycling. The null hypotheses were as follows: the bond strength values would not be affected by (1) the surface treatment method and (2) the aging protocol used.

\section{Materials and Methods}

The materials used in this study, with their commercial names and manufacturers, are shown in Table 1.

The present investigation used 5 CAD/CAM blocks of Vita Enamic. 10 specimens $(3 \times 8 \times 10 \mathrm{~mm})$ were prepared from the original blocks using a slow-speed diamond saw with water cooling (Mecatome T201A, Presi, France). One surface of each specimen was polished using silicon carbide paper (\#800, \#1000, \#1200, Buehler) under water spray. The specimens were then cleaned ultrasonically (Eurosonic 4D, Euronda, Italy) in distilled water for 5 minutes to remove any surface debris.

The polished surfaces of the blocks were conditioned with 5 different surface treatment methods:

1. Control(PA): the surfaces were etched with $35 \%$ phosphoric acid (Select HV Etch, Bisco, Inc.) for one minute and rinsed with distilled water for one minute.

2. Sandblasting (SB): the surfaces were air-abraded with $50-\mu \mathrm{m}$ of aluminum oxide particles from a distance of approximately $10 \mathrm{~mm}$ perpendicular to the treated surface at 4 bar pressure for 20 seconds.

3. Hydrofluoric acid (HF): the surfaces were etched with 9.5\% HF (Porcelain Etchant, Bisco, Inc., Schaumburg, IL) for 60 seconds and rinsed for one minute and then dried.

4. Er: YAG laser irradiation (L1): the Er:YAG laser (Pluser, Er:YAG doctor Smile, Italy) was used to irradiate the surfaces. A contact hand piece with a $600 \mu \mathrm{m}$ diameter was positioned perpendicular to the ceramic surface at a distance of $1 \mathrm{~mm}$ and moved manually with a sweeping motion over the entire surface for 10 seconds, and water (80\%) and air (70\%) were simultaneously sprayed to keep the surface cool. The laser irradiation of all the specimens was performed by the same operator. The laser-treatment parameters were as follows: pulse energy: $200 \mathrm{~mJ}$; power: $2 \mathrm{~W}$; pulse mode: $100 \mu$ s pulse duration; pulse rate: $10 \mathrm{~Hz}$; energy density of $7070.14 \mathrm{~J} / \mathrm{cm}^{2}$.

5. Er: YAG laser irradiation (L2): the surfaces were irradiated using different parameters of the Er: YAG laser, which were as follows: pulse energy: $300 \mathrm{~mJ}$;

Table 1. Materials used in this study ${ }^{19,20}$

\begin{tabular}{|c|c|c|c|}
\hline Material & Product & Composition & Manufacturer \\
\hline $\begin{array}{l}\text { Hybrid } \\
\text { ceramic }\end{array}$ & Vita Enamic & $\begin{array}{l}\text { Fine-structure feldspar ceramic: } 86 \% \text { wt } \\
\mathrm{SiO}_{2}, \mathrm{Al}_{2} \mathrm{O}_{3}, \mathrm{Na}_{2} \mathrm{O}, \mathrm{K}_{2} \mathrm{O}, \mathrm{B}_{2} \mathrm{O}, \mathrm{CaO}, \mathrm{TiO}_{2} \\
\text { Polymer: } 14 \% \text { wt } \\
\text { UDMA (urethane dimethacrylate) } \\
\text { TEGDMA (triethylene glycol dimethacrylate) }\end{array}$ & $\begin{array}{l}\text { VITA Zahnfabrik, Bad Sackingen, } \\
\text { Germany }\end{array}$ \\
\hline $\begin{array}{l}\text { Dual-cured } \\
\text { resin cement }\end{array}$ & $\begin{array}{l}\text { Duo-link } \\
\text { Universal }\end{array}$ & $\begin{array}{l}\text { Base: bis-GMA, triethyleneglycol dimethacrylate, urethane dimethacrylate, } \\
\text { glass filler } \\
\text { Catalyst: bis-GMA, triethyleneglycol dimethacrylate, glass filler }\end{array}$ & Bisco Inc, Schaumburg, IL, USA \\
\hline
\end{tabular}


power: $3 \mathrm{~W}$; pulse mode: 100 us pulse duration; pulse rate: $10 \mathrm{~Hz}$; energy density of $10615.71 \mathrm{~J} / \mathrm{cm}^{2}$.

The power of the laser was checked with a power meter before irradiation (laser point, Italy).

After all surface treatments, the ceramic blocks were cleaned in distilled water in an ultrasonic unit for 5 minutes and then air-dried for 60 seconds. A 2- part silane coupling agent (Bis-Silane, Bisco, Schaumburg, IL) was mixed and applied to the treated surface with a microbrush for 30 seconds according to the manufacturer's instruction and gently air-dried using oil-free compressed air. Two similarly treated surfaces were bonded together with dual-cured resin cement (Duo-link universal, Bisco Inc., Schaumburg, IL) according to the manufacturer's instructions. Excess luting cement was removed using a disposable micro-brush. The cemented blocks were submitted to a static load of $1 \mathrm{~kg}$ for 5 minutes, leaving the material to set in the self-curing modality. After that, the exposed cement was light-cured for 40 seconds from each side with an LED light curing unit (Bluephase, Ivoclar Vivadent, Schaan, Liechtenstein) at an irradiance level of $1100 \mathrm{~mW} / \mathrm{cm}^{2}$ to ensure optimal polymerization. The bonded specimens were stored in distilled water for 24 hours at $37^{\circ} \mathrm{C}$ prior to the $\mu$ TBS test.

The specimens were then embedded in acrylic resin blocks. After that, the specimens were vertically sectioned into serial slabs and further into steaks with approximately $1 \mathrm{~mm}^{2}$ cross-sectional areas using a slow-speed watercooled diamond saw in a cutting machine (Mecatome T201A, Presi, France). Thirty-two beams were obtained from each block. The peripheral slices were excluded, as the result could be influenced by either an excess or a deficient amount of resin cement at the interface. Half of the specimens were tested immediately and the other half of beams were tested after thermocycling (TC) $\left(6000\right.$ cycles, $\left.5^{\circ} \mathrm{C}-55^{\circ} \mathrm{C}\right)$; the dwell time and the transfer time were 30 seconds and 10 seconds respectively. The specimens were glued to the grips of a microtensile testing machine (Microtensile tester, Bisco, Germany) using a cyanoacrylate adhesive and tested in tension at a crosshead speed of $0.5 \mathrm{~mm} / \mathrm{min}$ until failure. The crosssectional areas of the specimens at the fracture site were measured with a digital caliper (Mitutoyo, Japan) to calculate the tensile bond strength in MPa. All fractured specimens were evaluated by a stereomicroscope (Leica EZ4D, Leica Microsystems Ltd, Switzerland; ×40) to determine the fracture pattern. The failure modes were classified as type 1: adhesive failure (Vita Enamic surface was visible), type 2: mixed failure in Vita Enamic and luting resin cement (failure line includes both ceramic and resin luting cement), Type 3: a cohesive fracture in resin luting cement. Representative specimens of each group were observed by a scanning electron microscope. The specimens were rinsed with $96 \%$ ethanol and air-dried, fixed on metallic stubs, sputter-coated with a gold layer, and then examined under a scanning electron microscope
(Philips XL30, Netherlands) at $\times 300$ magnification.

Statistical Analysis

Using the Kolmogorov-Smirnov test, the normality of data distribution was tested. $(P>0.05)$. The bond strength of each group was analyzed by two-way analysis of variance (ANOVA) to determine the significant differences between the surface treatment methods and thermo-cycling. Tukey HSD comparison tests were used for analyzing the $\mu \mathrm{TBS}$ data in MPa. For all analyses, a significance level of 0.05 and a confidence level of $95 \%$ were considered. IBM SPSS 22 was used for analysis.

\section{Results}

The mean and standard deviations of $\mu$ TBS data (MPa) are presented in Figure 1. There was no significant interaction between surface treatment methods and thermocycling $(P=0.671)$. Two-way ANOVA of the $\mu$ TBS values revealed that the bond strength was significantly affected by the surface treatment method $(P<0.001)$. All the specimens submitted to thermocycling showed lower bond strength values which were significantly different from those not submitted to thermo-cycling $(P=0.007)$.

The highest $\mu$ TBS values in non-thermal and thermal aged groups belonged to the HF group. It was followed by the SB and PA groups and the bond strength values for the former was significantly higher than the latter $(P=0.019)$. All laser treated specimens showed the weakest bond strength. The $\mu$ TBS values of the two laser groups, accompanied by an aging procedure or not, were not statistically different. Table 2 gives the modes of failure. The SEM images of the fracture surfaces of nonthermocycled specimens are presented in Figure 2.

\section{Discussion}

Vita Enamic, a hybrid ceramic with a dual network structure, has been recently introduced as an indirect esthetic restorative material. ${ }^{21,22}$ As bonding of indirect

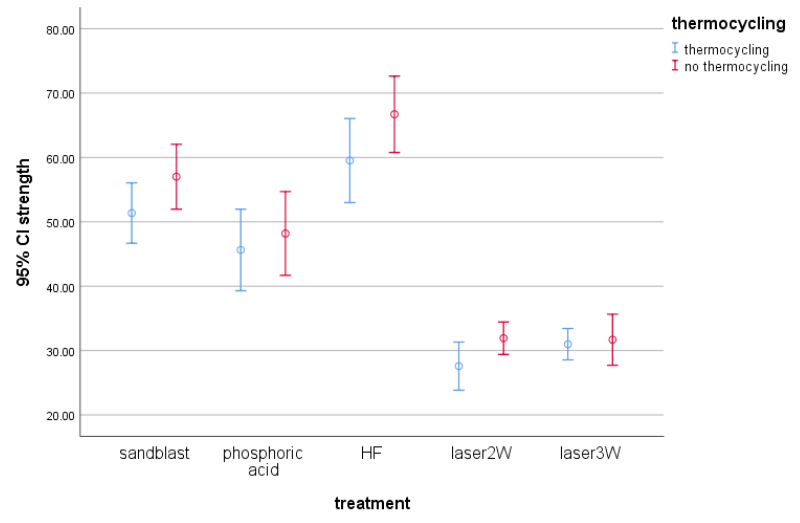

Figure 1. Mean $\mu \mathrm{TBS}$ (MPa) Results as a Function of the Type of Surface Treatment and Thermocycling. The vertical bars denote 0.95 confidence intervals. 
Table 2. Percentage Distribution of Failure Modes

\begin{tabular}{|c|c|c|c|c|c|c|c|c|c|c|c|c|c|c|c|}
\hline \multirow[b]{3}{*}{ Aging protocol } & \multicolumn{15}{|c|}{ Surface Treatment Methods } \\
\hline & \multicolumn{3}{|c|}{ PA } & \multicolumn{3}{|c|}{$\mathrm{HF}$} & \multicolumn{3}{|c|}{ SB } & \multicolumn{3}{|c|}{ ER:YAG 2W } & \multicolumn{3}{|c|}{ ER:YAG 3W } \\
\hline & 1 & 2 & 3 & 1 & 2 & 3 & 1 & 2 & 3 & 1 & 2 & 3 & 1 & 2 & 3 \\
\hline Non-thermocycled & 13 & 3 & 0 & 1 & 12 & 3 & 11 & 5 & 0 & 6 & 10 & 0 & 5 & 11 & 0 \\
\hline Thermocycled & 15 & 1 & 0 & 2 & 13 & 1 & 9 & 7 & 0 & 6 & 10 & 0 & 1 & 15 & 0 \\
\hline
\end{tabular}

restorations is highly dependent on their adhesive interface and essential to ensure a favorable outcome, this in vitro study was designed to examine the impact of different surface treatment methods on the adhesion of a dualcured resin cement to Vita Enamic and to assess the bond durability after thermocycling. Regarding the surface treatments, it could be observed that this factor affected the $\mu$ TBS values and varied among different groups; thus, the first hypothesis about the ineffectiveness of the type of surface conditioning on the bond strength values was rejected. In addition, thermocycling significantly decreased the bond strength; thus, the second hypothesis regarding insignificant influence of the aging protocol on the bond strength values was also rejected.

The etchability of the ceramic or ceramic-like material is an important part of the information required for the clinician to indicate the way the restoration is luted. ${ }^{23}$ Enamic is a material consisting of a dominant ceramic network infiltrated with a monomer mixture..$^{21,22}$ In this respect, as could be expected in the present study, the ceramic content of the hybrid material guided the surface treatment and the hybrid ceramic specimens etched by hydrofluoric acid attained the bond strength values higher than the specimens treated with other methods. The glass content of the feldspar ceramic network is dissolved in $\mathrm{HF}$ and also part of the polymer, ${ }^{22}$ producing an irregular and retentive surface. Through silanization, the surface is conditioned to bond to the resin matrix of the resin luting cement and provide an effective interaction. ${ }^{11,18}$ Using the silane coupling agent with silica based ceramics is an essential factor to increase the wettability of the surface and create a hydrophobic surface for improving a chemical bond to the resin cement. ${ }^{18}$ These might explain the higher bond strength achieved with this treatment method. The HF and silanization caused the specimens to fail predominantly in a mixed mode (Table 2, Figure 2B). In this study, silane was applied to the treated surfaces in all groups to obtain similar preparation regarding the influence of the silane coupling agent.

Air-abrasion by pure alumina is a method of surface treatment for an indirect composite and high strength ceramics. $^{24,25}$ Surface preparation by abrasive blasting is assumed to be different depending on the setting of a wide range of parameters such as particle size, pressure, distance from the surface, working time, and impact angle. ${ }^{26}$ It increases surface energy, wettability, surface roughness and irregularities which may augment the interlocking between the resin cement and the ceramic. ${ }^{27,28}$ In the current study, the $\mu$ TBS of the sandblasting surface treatment group was lower than that of the hydrofluoric acid treatment group. The fractured surface of the SB specimen examined by SEM
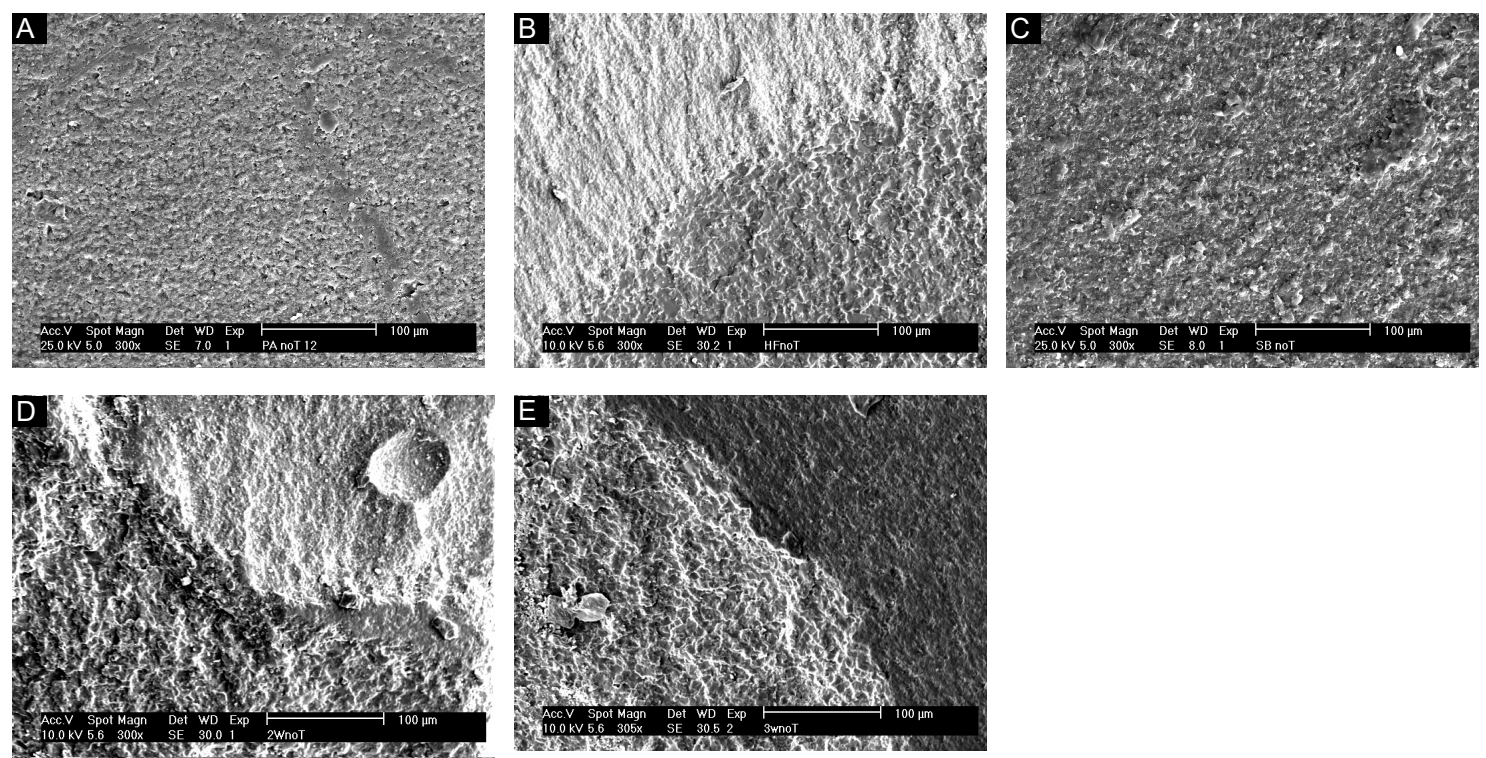

Figure 2. Representative SEM Micrograph $(\times 300)$ of debonded surfaces of no-thermocycled specimens. A: Phosphoric acid (PA); B: Hydrofluoric acid (HF); C: Sand blasting(SB); D: 2W Er:YAG laser; E: 3WEr:YAG laser. 
also revealed a relatively homogenous surface not deeply dented (Table 2, Figure 2C), which might explain the lower bond strength values compared with the HF group. Hydrofluoric acid chemically reacts with the silica phase of the fine-structured feldspathic component ${ }^{29}$ of VE and penetrates into the depth of the hybrid ceramic rather than the merely surface alteration made by sandblasting. ${ }^{29}$ Consequently, HF might create more significant microporosities for mechanical interlocking with the resin luting cement. Kern and Thompson have stated that sandblasting of feldspathic ceramics should be avoided since it can cause a huge volume loss in the materials. ${ }^{30}$ Also, it has been stated that although sandblasting increases surface roughness, such irregularities are not in the form of retentive undercuts formed by hydrofluoric acid treatment, ${ }^{31}$ and the undercuts with sharp angles may impede the complete wetting of the surface which could adversely affect the bond strength. ${ }^{32}$ Yoshihara et al showed that sandblasting caused a crack on the surface of all the investigated composite CAD/CAM block. ${ }^{33}$ Tekçe et al also reported lower values of micro-tensile bond strength of Vita Enamic to dual-cured resin cement after surface sandblasting for 60 seconds in comparison with a shorter period of air abrasion. The adhesion stability after 5000 thermo-cycles was adversely affected by prolonged sandblasting. ${ }^{34}$

The finding of the current study is in agreement with other investigations ${ }^{11,35}$ which found the HF treatment and silanization method as the most effective treatment approach for improving the strength of the bond between the hybrid ceramic and resin cement in comparison with the air abrasion method.

One surface treatment method used in the present study consisted only of etching with phosphoric acid. It was a more effective procedure to obtain higher bond strength values in comparison with laser irradiation but it led to significantly lower values than those in the HF and SB treatment groups. Etching with phosphoric acid is a routine step in adhesive procedures. Although phosphoric acid is not capable of modifying the surface topography, ${ }^{11}$ it has a cleansing effect, eliminates the surface debris and enhances surface energy and wettability of the surface. ${ }^{11,36}$ The subsequent application of a silane coupling agent might have improved the union between the surface of the hybrid ceramic and the resin cement. It can be assumed that adequate surface activation was provided through silanization and chemical interaction rather than micromechanical bonding. ${ }^{37}$ This might be explained by the failure mode which was mainly adhesive (Table 2, Figure 2A).

The other method of surface treatment used in this study was Er:YAG laser etching with two different output powers. The low power setting was considered for the current study to avoid damage induced by heat to the surface of the laser irradiated ceramic specimens. Gökçe et $\mathrm{al}^{13}$ demonstrated that the highest strength of the bond to lithia-based ceramic could be achieved with a low Er:YAG power setting at $300 \mathrm{~mJ}$ in comparison to 600 and $900 \mathrm{~mJ}$ and it was attributed to the fact that a high laser power setting could be destructive to the porcelain surface through heating damage and dissociation of the crystal and/or matrix phases. Laser irradiation creates a rough surface by different mechanisms such as removing the glass phase of the porcelain ${ }^{13}$ or through the ablation of the resin matrix ${ }^{38}$ and the explosion of the flowing water spray ${ }^{16}$ In the current study, laser etching caused the lowest bond strength regardless of the power setting. The low bond strength could be attributed to two factors. Firstly, it could be related to the low energy density of the irradiated laser which might result in shallow irregularities and less defined penetration zone of the luting resin cement. ${ }^{39}$ Secondarily, the heat could damage the superficial layer of the hybrid ceramic following laser irradiation which might be separated from the underlying intact ceramic during bond strength test, while the outermost layer remained interlocked with the resin luting cement. ${ }^{13}$ Several studies have concluded that Er:YAG laser etching has been inadequate for improving the strength of the bonds of feldspathic porcelain to the resin cement ${ }^{39}$ or to the resin composite. ${ }^{40}$ Mixed failures were the most prevalent failure type in laser irradiated specimens. (Table 2, Figure 2D,2E)

The bonding interface could be affected in a humid and thermally dynamic oral environment. In the current study, thermocycling was used to artificially age the adhesive interface for assessing the stability of the resin cement interaction with the hybrid ceramic. After 6000 thermocycling, the specimens exposed to the aging protocol exhibited significantly lower bond strength in all groups in comparison to the non-aged specimens. Similarly, Lise et al found lower bond strength values of Vita Enamic specimens to self-adhesive cement and a light-curing flowable resin composite following water storage for 6 months regardless of the type of the surface treatment for the CAD/CAM blocks. ${ }^{12}$ This could be due to the penetration of small water molecules into small spaces at the adhesive interface and between polymer chains, resulting in decreased stability of the union and causing its plasticization. ${ }^{9,11}$

Considering the limitation of this study, it is recommended that the hybrid ceramic, Vita Enamic, should be treated similarly to an etchable silica/glassbased systems since HF application and silanization provided the highest bond strength. However, it is clear that other brands of hybrid ceramics and resin cements need to be tested to provide more reliable treatment protocols for hybrid ceramics. In addition, in order to use an Er:YAG laser for superficial treatment of hybrid ceramics, more studies are still required to examine the different parameters of such lasers and their possible effects. 


\section{Conclusion}

Based on the results obtained and within the limitations of this in vitro study, it was concluded that the use of hydrofluoric acid was the most effective surface treatment method for the hybrid ceramic to provide the highest strength of the bond to the luting resin cement. Sandblasting of the hybrid ceramic with $50 \mu \mathrm{m} \mathrm{Al}_{2} \mathrm{O}_{3}$ particles was not effective in the HF surface conditioning method to improve the bond strength values. Acid etching with phosphoric acid revealed higher bond strength values in comparison to Er:YAG laser etching. The Er:YAG laser conditioning method within the parameters tested showed lower bond strength compared to the other surface treatment methods. Thermo-cycling showed a considerable influence on the hybrid ceramic/luting resin cement interface and caused a significant reduction in the bond strength values after all surface treatment methods.

\section{Ethical Considerations}

Not applicable.

\section{Conflict of Interests}

The authors declare no conflict of interest.

\section{References}

1. Della Bona A. Bonding to ceramics: scientific evidences for clinical dentistry. Sao Paulo: Artes Medicas; 2009.

2. Kelly JR. Ceramics in restorative and prosthetic dentistry. Annu Rev Mater Res. 1997;27(1):443-468.

3. Ferracane JL. Resin composite--state of the art. Dent Mater. 2011;27(1):29-38. doi:10.1016/j.dental.2010.10.020

4. Petrini M, Ferrante M, Su B. Fabrication and characterization of biomimetic ceramic/polymer composite materials for dental restoration. Dent Mater. 2013;29(4):375-381. doi:10.1016/j.dental.2012.12.004

5. Dirxen C, Blunck U, Preissner S. Clinical performance of a new biomimetic double network material. Open Dent J. 2013;7:118-122. doi:10.2174/1874210620130904003

6. Lawson NC, Bansal R, Burgess JO. Wear, strength, modulus and hardness of CAD/CAM restorative materials. Dent Mater. 2016;32(11):e275-e283. doi:10.1016/j. dental.2016.08.222

7. Stamatacos C, Simon JF. Cementation of indirect restorations: an overview of resin cements. Compend Contin Educ Dent. 2013;34(1):42-44, 46.

8. Thompson JY, Stoner BR, Piascik JR, Smith R. Adhesion/ cementation to zirconia and other non-silicate ceramics: where are we now? Dent Mater. 2011;27(1):71-82. doi:10.1016/j.dental.2010.10.022

9. Elsaka SE. Bond strength of novel CAD/CAM restorative materials to self-adhesive resin cement: the effect of surface treatments. J Adhes Dent. 2014;16(6):531-540. doi:10.3290/j.jad.a33198

10. Spitznagel FA, Horvath SD, Guess PC, Blatz MB. Resin bond to indirect composite and new ceramic/polymer materials: a review of the literature. J Esthet Restor Dent. 2014;26(6):382-393. doi:10.1111/jerd.12100

11. Campos F, Almeida CS, Rippe MP, de Melo RM, Valandro LF, Bottino MA. Resin bonding to a hybrid ceramic: effects of surface treatments and aging. Oper Dent. 2016;41(2):171178. doi:10.2341/15-057-1

12. Lise DP, Van Ende A, De Munck J, Vieira L, Baratieri LN, Van Meerbeek B. Microtensile bond strength of composite cement to novel CAD/CAM materials as a function of surface treatment and aging. Oper Dent. 2017;42(1):73-81. doi:10.2341/15-263-1

13. Gökçe B, Özpinar B, Dündar M, Cömlekoglu E, Sen B, Güngör M. Bond strengths of all-ceramics: acid vs laser etching. Oper Dent. 2007;32(2):173-178. doi:10.2341/06-52

14. Yavuz T, Dilber E, Kara HB, Tuncdemir AR, Ozturk AN. Effects of different surface treatments on shear bond strength in two different ceramic systems. Lasers Med Sci. 2013;28(5):1233-1239. doi:10.1007/s10103-012-1201-5

15. de Paula Eduardo C, Bello-Silva MS, Moretto SG, Cesar PF, de Freitas PM. Microtensile bond strength of composite resin to glass-infiltrated alumina composite conditioned with Er, Cr: YSGG laser. Lasers Med Sci. 2012;27(1):7-14. doi:10.1007/s10103-010-0822-9

16. Bader C, Krejci I. Indications and limitations of Er: YAG laser applications in dentistry. Am J Dent. 2006;19(3):178186.

17. Barutcigil K, Barutcigil Ç, Kul E, Özarslan MM, Buyukkaplan US. Effect of different surface treatments on bond strength of resin cement to a CAD/CAM restorative material. J Prosthodont. 2019;28(1):71-78. doi:10.1111/ jopr.12574

18. Blatz MB, Sadan A, Kern M. Resin-ceramic bonding: a review of the literature. J Prosthet Dent. 2003;89(3):268274. doi:10.1067/mpr.2003.50

19. Egbert JS, Johnson AC, Tantbirojn D, Versluis A. Fracture strength of ultrathin occlusal veneer restorations made from CAD/CAM composite or hybrid ceramic materials. Oral Sci Int. 2015;12(2):53-58. doi:10.1016/S13488643(15)00017-8

20. Onay EO, Korkmaz Y, Kiremitci A. Effect of adhesive system type and root region on the push-out bond strength of glass-fibre posts to radicular dentine. Int Endod J. 2010;43(4):259-268. doi:10.1111/j.1365-2591.2009.01643.x

21. Della Bona A, Corazza PH, Zhang Y. Characterization of a polymer-infiltrated ceramic-network material. Dent Mater. 2014;30(5):564-569. doi:10.1016/j.dental.2014.02.019

22. Duarte S, Sartori N, Phark JH. Ceramic-reinforced polymers: CAD/CAM hybrid restorative materials. Curr Oral Health Rep. 2016;3(3):198-202. doi:10.1007/s40496016-0102-2

23. Gracis S, Thompson VP, Ferencz JL, Silva NR, Bonfante EA. A new classification system for all-ceramic and ceramiclike restorative materials. Int J Prosthodont. 2015;28(3):227235. doi:10.11607/ijp.4244

24. D’Arcangelo C, Vanini L. Effect of three surface treatments on the adhesive properties of indirect composite restorations. J Adhes Dent. 2007;9(3):319-326.

25. Kern M. Resin bonding to oxide ceramics for dental restorations. J Adhes Sci Technol. 2009;23(7-8):1097-1111. doi:10.1163/156856109X432721

26. Aswal GS, Nair CK. Effects of various parameters of alumina air abrasion on the mechanical properties of low fusing feldspathic porcelain laminate material. $S A D J$. 2015;70(4):150-155.

27. Kirmali O, Barutcugil C, Harorli O, Kapdan A, Er K. 
Resin cement to indirect composite resin bonding: effect of various surface treatments. Scanning. 2015;37(2):89-94. doi:10.1002/sca.21183

28. Sen D, Poyrazoglu E, Tuncelli B, Goller G. Shear bond strength of resin luting cement to glass-infiltrated porous aluminum oxide cores. J Prosthet Dent. 2000;83(2):210-215.

29. Moravej-Salehi E, Moravej-Salehi E, Valian A. Surface topography and bond strengths of feldspathic porcelain prepared using various sandblasting pressures. $J$ Investig Clin Dent. 2016;7(4):347-354. doi:10.1111/jicd.12171

30. Kern M, Thompson VP. Sandblasting and silica coating of a glass-infiltrated alumina ceramic: volume loss, morphology, and changes in the surface composition. $J$ Prosthet Dent. 1994;71(5):453-461.

31. Valian A, Moravej-Salehi E. Surface treatment of feldspathic porcelain: scanning electron microscopy analysis. J Adv Prosthodont. 2014;6(5):387-394. doi:10.4047/ jap.2014.6.5.387

32. Amaral R, Özcan M, Bottino MA, Valandro LF. Microtensile bond strength of a resin cement to glass infiltrated zirconia-reinforced ceramic: the effect of surface conditioning. Dent Mater. 2006;22(3):283-290. doi:10.1016/j.dental.2005.04.021

33. Yoshihara K, Nagaoka N, Maruo Y, Nishigawa G, Irie M, Yoshida Y, et al. Sandblasting may damage the surface of composite CAD-CAM blocks. Dent Mater. 2017;33(3):e124-e135. doi:10.1016/j.dental.2016.12.003
34. Tekce N, Tuncer S, Demirci M. The effect of sandblasting duration on the bond durability of dual-cure adhesive cement to CAD/CAM resin restoratives. J Adv Prosthodont. 2018;10(3):211-217. doi:10.4047/jap.2018.10.3.211

35. Frankenberger R, Hartmann VE, Krech M, Krämer N, Reich S, Braun A, et al. Adhesive luting of new CAD/CAM materials. Int J Comput Dent. 2015;18(1):9-20.

36. Wendler M, Belli R, Panzer R, Skibbe D, Petschelt A, Lohbauer U. Repair Bond Strength of Aged Resin Composite after Different Surface and Bonding Treatments. Materials (Basel). 2016;9(7). doi:10.3390/ma9070547

37. Della Bona A, Anusavice KJ, Hood JA. Effect of ceramic surface treatment on tensile bond strength to a resin cement. Int J Prosthodont. 2002;15(3):248-253.

38. Correa-Afonso AM, Palma-Dibb RG, Pecora JD. Composite filling removal with erbium:yttrium-aluminum-garnet laser: morphological analyses. Lasers Med Sci. 2010;25(1):17. doi:10.1007/s10103-008-0581-z

39. Shiu P, de Souza-Zaroni WC, de Paula Eduardo C, Youssef MN. Effect of feldspathic ceramic surface treatments on bond strength to resin cement. Photomed Laser Surg. 2007;25(4):291-296. doi:10.1089/pho.2007.2018

40. Akyıl MŞ, Yilmaz A, Karaalioğlu OF, Duymuş ZY. Shear bond strength of repair composite resin to an acidetched and a laser-irradiated feldspathic ceramic surface. Photomed Laser Surg. 2010;28(4):539-545. doi:10.1089/ pho.2009.2586 